\title{
INTERACTION BEYOND THE MAIN SEQUENCE: ONE RECIPE?
}

\author{
Jean-Pierre De Greve \\ Astrophysical Institute \\ Vrije Universiteit Brussel \\ Pleinlaan 2, B-1050 Brussels \\ Belgium
}

\begin{abstract}
We investigate the different aspects that govern the interaction of post-main-sequence evolution of binaries, using a new, homogeneous set of computations. The set describes the evolution of both components through the phase of mass transfer, till the end of the core helium burning of the primary. The mass range is 9 to $40 \mathrm{M}_{0}$, the mass ratios are 0.9 and 0.6 (and introducing 0.99 as a newcomer). Both for small and large masses we discuss the consequences of non-conservative mass transfer. Using a simple concept for the fraction $B$ of transferred matter, we look to its value at the onset of mass transfer for various mass ratios and periods. Exploration of the phase space of interacting binaries reveals the influence of the various parameters on the dimensions of the resulting systems after mass transfer. Special attention is given to binaries with mass ratios very close to one. Their secondaries evolve directly into yellow supergiants such as observed in the LMC.
\end{abstract}

\section{Introduction : some old stuff, still badly known.}

Describing the effect of gravitational interaction by means of mass transfer from one component to the other, forms the basis of our understanding of the formation of a lot of different systems : WR-binaries, Algols, cataclysmics, etc. Most of the advanced binaries with periods of the order of days or shorter, are believed to have evolved through one or another kind of mass transfer.

Now, when we look to that extended mass range involved, the question arises whether we can adopt a single model of mass transfer for small as well as for large masses.

Let us first notice that internal structure alone is already responsable for a gradual change in the resulting characteristics of the system and its components for increasing primary mass. Once the gravitational instability exists, the resulting mass transfer will stop only when the amount of internally produced energy exceeds a treshold value large enough to stop core contraction, and when the outer layers become more transparant to lose the excess of energy more efficiently. This means a combination of core helium burning with a deficient hydrogen abundance of the outer layers (typically $X_{a t}=0.2-0.3$ ). The value of the remnant mass of the loser is therefore basicly rellated to the frozen-in hydrogen profile, and hence, to the original convective core mass.

Indeed, the results of computations of case B mass transfer, given in Figure 1, confirm that the relative growth of the ratio of the remnant mass to the initial mass is nothing else but a measure of the relative growth of the ratio of mass of the convective core with initial mass. 
Therefore, the predominance of high mass ratios among the massive Algols, noticed by Giuricin and Mardirossian (1981), and early-type sd systems (Hilditch and Bell, 1987 ; Bell et al., 1991), is not necessarily a consequence of a different formation mechanism, as was already argued earlier (De Greve, 1986).

Other effects are of much less importance. The calculations by Huang and Taam (1990) for a massive system of $40 \mathrm{M}_{0}+25 \mathrm{M}_{\mathrm{o}}$ show that neither irradiation nor tidal effects lead to an enhanced mass loss, although the results depend on the form of the empirical mass loss formula and on the period of the system. These authors also demonstrate that opacity effects are responsable for the non-application of radiation pressure lobes during the mass transfer.

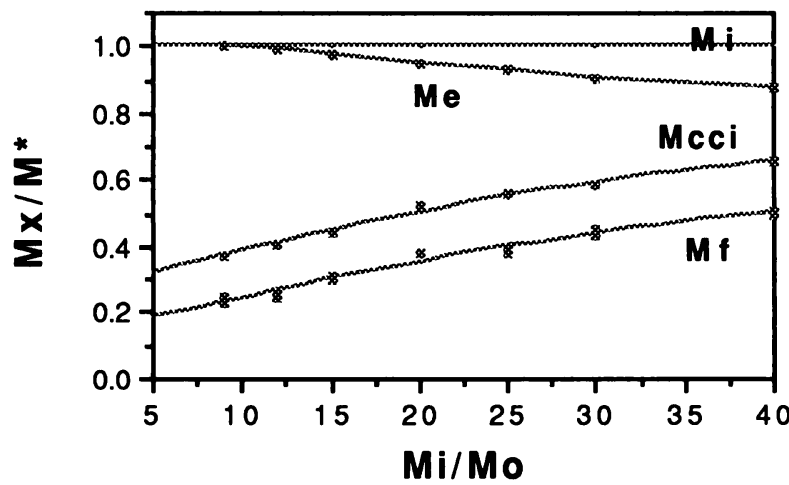

Figure 1. Specific mass values of primary components in close binary systems (normalized to the initial mass), as a function of the initial mass of the primary. Mi: initial mass; Me: mass at the end of core hydrogen burning; Mcci: mass of the initial convective core; Mf: remanant mass after a case B of mass transfer.

\section{Mass loss from the system depending on $q$}

During RLOF, both the timescale of mass loss from the primary and of the accretion by the secondary are dictated by the thermal timescales of the components. Tutukov and Yungelson (1991) therefore used the ratio of these timescales at the onset of RLOF, to describe the efficiency of the mass transfer. After introducing the Roche radii as approximations for the radii, and a mass-luminosity relation for massive stars (Figuereido et al., 1991), Vrancken et al. (1991) derived the following relation

$$
\beta=\left(M_{2} / M_{1}\right)^{1.84}
$$

for the mass range $10-30 \mathrm{M}_{0}$.

For lower masses $\left(5 \mathrm{M}_{\mathrm{o}}<\mathrm{M}_{\mathrm{i}}<15 \mathrm{M}_{0}\right.$ ), with a different mass-luminosity relation (Griffiths et al., 1988, $\mathrm{L} \sim \mathrm{M}^{3.51}$ ), we obtain

$$
\beta=\left(M_{2} / M_{1}\right)^{2.95}
$$


Figure 2 gives the value of $B$ for different $q$-values. As can be seen, the simple model leads to increasing nonconservative mass transfer with decreasing mass ratio. There is also an overall decrease for lower masses, although the differences remain small.

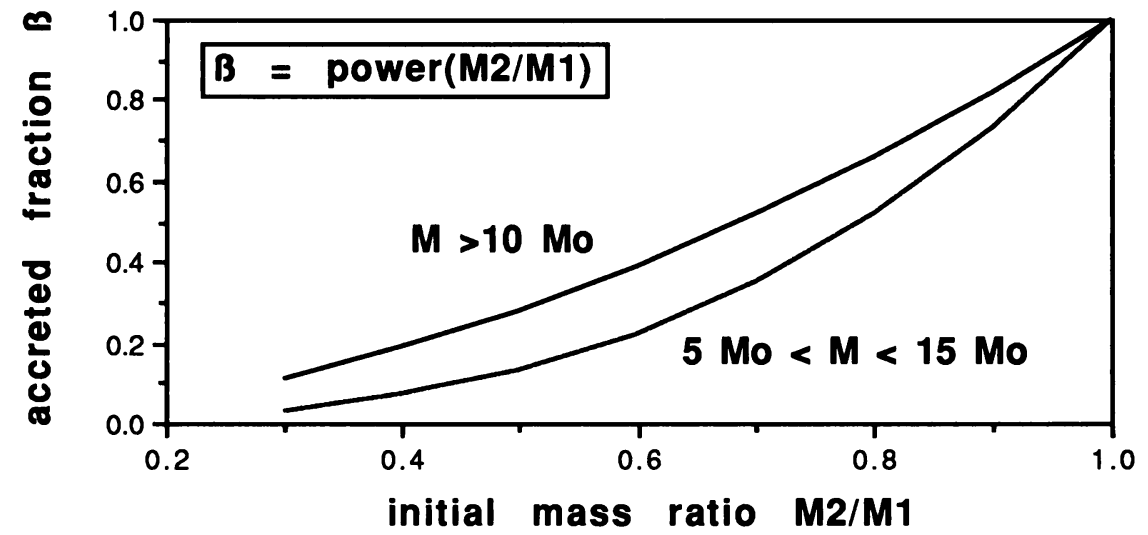

Figure 2. Fraction $B$ of accreted mass during a case $B$ of mass transfer as a function of the initial mass ratio, adopting a power law of that mass ratio (see text).

A better approximation is obtained if we use the real values of the stellar parameters at the onset of RLOF. In such a way, the variation of $B$ across the HRD can be shown (Figure 3 ). Hence, the simple assumption of accretion proportional to the ratio of the thermal timescales predicts a large mass loss from the system for small mass ratios, for low effective temperatures, and for low masses.

Nevertheless, the effect of this on the final period cannot be used as a test, because the periods all converge towards small values, when a common envelope algorithm is used for the period variation (Yungelson and Tutukov, 1991 ; Vrancken et al. 1991). Apparently, this contradicts the observations of long period WR systems and Algols. An answer may be found in the suggestion by Livio and Soker (1988), that more evolved supergiants, with more centrally condensed configurations, spin up more rapidly, thus slowing down the orbital decay. But the situation is more complex, because also the energy transport to the surface may be more efficient, increasing the luminosity of the star. Finally, the common envelope scenario may not at all be applicable to early and mid case B interaction.

\section{Accretion onto the secondary :}

Accretion has 3 main effects on the secondary component :

a) it makes it more massive, hence brighter and evolving faster,

b) it spins up the outer layers 
c) it changes the chemical composition of the outer layers when nuclear burnt matter is transferred.

When the secondary has a convective core, a fourth effect, the rejuvenation through convective core growth, comes into play.

Clearly, the amount of these effects depend on $B$.

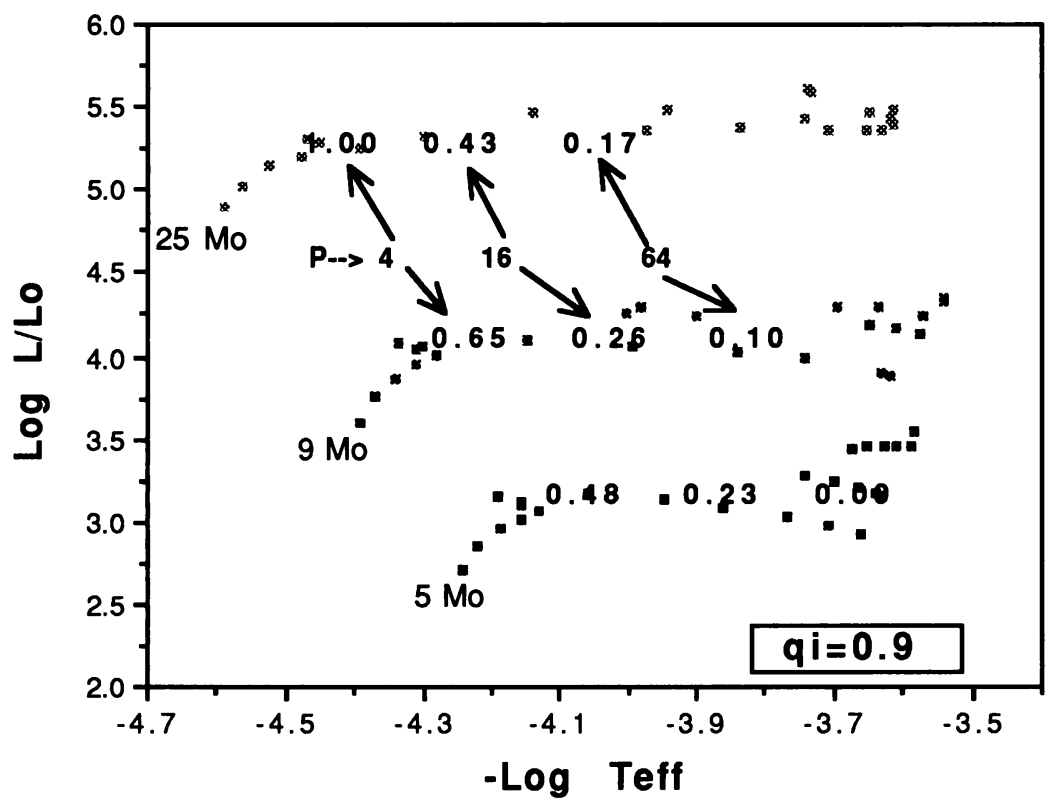

Figure 3. Fraction $B$ of accreted matter during a case $B$ of mass transfer, expressed as the ratio of the Kelvin-Helmholtz timescales of primary to secondary companent at the onset of the mass transfer, for an initial mass ratio of 0.9 and various initial periods.

The spin up by accretion has not been studied in much detail, mainly because it's only worth doing it hydrodynamically. However, both assuming rigid rotation or differential rotation leads to a fast spin up towards the rotational break-up velocity (Packet, 1988). The results, obtained by Packet, using a simple model of a rigid rotator with constant radius, accreting mass from a Keplerian accretion disk, show that for systems with periods of the order of several days and more, the break-up velocity is reached after only a small fraction of mass is accreted.

Van Hamme and Wilson (1990) investigated the rotation statistics of 36 Algol type binaries. They found a subset rotating at centrifugal limit. They also conclude that many Algols have sufficient mass transfer to maintain nonsynchronous rotation, although they do not reach the centrifugal limit (RY Gem rotates at 14 times $v_{\text {sync, }}$, whereas $v_{\text {centrif. }}=$ $\left.24 \mathrm{v}_{\text {sync }}\right)$. Certainly this requires further work on the theory of differential rotation.

Does accretion stop as a result of rotational spin up ? Paczynski (1991), using a polytropic model for the three components (disk, boundary layer, star), found that all 
models, rotating at subcritical speed, accrete mass and angular momentum. In supercritical models, the angular velocity decreases monotically throughout the whole disk-star system. Popham and Narayan (1991) studied the same problem, and found that with increasing angular momentum, the spin-up slows down and eventually stops near break-up, allowing accretion to continue.

\section{To mix or not to mix.}

Transfer of matter goes on till the atmospheric hydrogen abundance of the loser reaches 0.3 to 0.2 . The affected matter is (partly) accreted by the secondary. What is the evidence that this matter is mixed in and what is its influence on the evolution?

a) Packet (1988) showed that simple accretion without mixing leads to HRD positions that are too hot and too small compared to the observations of semidetached or postRLOF systems (see also Proffitt and Michaud, 1989).

b) Bright components in semidetached systems have been observed having a underabundance of carbon, compared to the galactic value in the solar neighbourhood. There is a tendency for larger anomalies with smaller mass-ratios (De Greve and Cugier, 1989 , and posters in this volume).

The explanation is given by the so-called 'thermohaline mixing', a gradual mixing triggered by a Raleigh-Taylor instability as a result of a density inversion. Its functionality in a stellar structure context was examined by Kippenhahn et al. (1980), who found the timescale of the mixing to be shorter than the thermal timescale.

The detoriation of outer layers of bright components in binaries by mass transfer is also forwarded as an explanation of the apparent excess of post main sequence supergiants in the LMC. Compared to normal evolutionary tracks, the supergiant region in the LMC is well populated, especially the so-called "gap" between $\log$ Teff $=4.5$ and 4.3 (Fitzpatrick and Garmany, 1990). Ray and Rathnasree (1991) argue that a large fraction of them (60 $\%$ ) may occur in binaries. An enrichment of He in the outer layers slows down the evolutionary speed through the supergiant region (Tuchman and Wheeler, 1990). In their analysis, Ray and Rathnasree use a $20 \mathrm{M}_{0}$ star with $\mathrm{Y}=0.5$ in the outer $1.4 \mathrm{M}_{\mathrm{o}}$ of the 14 $\mathbf{M}_{\mathrm{o}}$ envelope. Their solution has implications on the number and orbital period distribution of massive X-ray binaries in the LMC.

Although helium enrichment thus apparently poses a natural explanation for the supergiant excess and has already some observational support (Kudritzki et al., 1989), it also raises a number of questions. Is the evolution in the LMC different from our Galaxy? Do actual models of close binary evolution support this idea ? In other words, how big is the effect of the mixing as a result of interaction?

Mass transfer stops when $X_{\mathrm{at} 1}=0.20-0.30$, hence the amount of transferred matter with deficient hydrogen abundance is

$$
\Delta \mathrm{M}_{\mathrm{d}}=\mathrm{M}_{\mathrm{cci}}-\mathrm{M}_{\mathrm{cc}}\left(\mathrm{X}_{\mathrm{c}}=0.2\right)=\Delta \mathrm{M}_{\mathrm{d}}=\mathrm{M}_{\mathrm{cci}}-\mathrm{M}_{\mathrm{f}}
$$

Of this, $B \Delta M_{d}$ is accreted by the gainer, which at that time has a mass of

$$
M_{2}=M_{2 e}+B\left(M_{1 e}-M_{c c i}\right)
$$


where index e indicates the value at the onset of mass transfer (taking into account stellar wind mass loss).

Because the frozen-in profile of $\mathrm{X}$ has a rather steep gradient, the dominant mass fraction in the accreting star contributing to the mixing, is the homogeneous outer mantle. Its mass is given by

$$
\Delta \mathbf{M}_{\mathrm{h}}=\mathrm{M}_{2}-\mathrm{M}_{\mathrm{cc}}\left(\mathrm{M}_{2}\right)
$$

Hence the result of the mixing is, in first approximation

$$
\mathrm{X}_{\mathrm{at}}=\frac{\Delta \mathrm{M}_{\mathrm{h}} \times 0.7+\Delta \mathrm{M}_{\mathrm{d}} \mathrm{x}<\mathrm{X}>}{\Delta \mathrm{M}_{\mathrm{h}}+\Delta \mathrm{M}_{\mathrm{d}}}
$$

$<\mathrm{X}>$ being the average value of $\mathrm{X}$ in the varying region that is transferred. Assuming a linear decrease of $\mathrm{X}$ with mass, resulting from the retreated convective core, and taking 0.2 as lower value, we may write $\langle\mathrm{X}\rangle=0.45$.

Hence, $\mathrm{Xat}=\frac{0.7 \Delta \mathrm{M}_{\mathrm{h}}+0.45 \Delta \mathrm{M}_{\mathrm{d}}}{\Delta \mathrm{M}_{\mathrm{h}}+\Delta \mathrm{M}_{\mathrm{d}}}$

Using best fit approximations for $\mathbf{M}_{\mathrm{cc}}, \mathrm{M}_{\mathrm{e}}$ and $\mathrm{M}_{\mathrm{f}}$ from the evolutionary computations of de Loore and De Greve (poster, this volume), we obtain the results shown in Figure 4. They show that thermohaline mixing does not lead to a severe helium enrichment in the outer layers.

Let us now look at the further evolution somewhat closer.

After thermohaline mixing, the models have a large homogeneous envelope, with a small transition zone towards the convective core. In order to reach that zone, 40 to $50 \%$ of the mass has to be removed. Rotation, even close to the break-up velocity, cannot account for a large enough increase of the stellar wind mass loss, to remove this mass during the remaining main sequence lifetime.

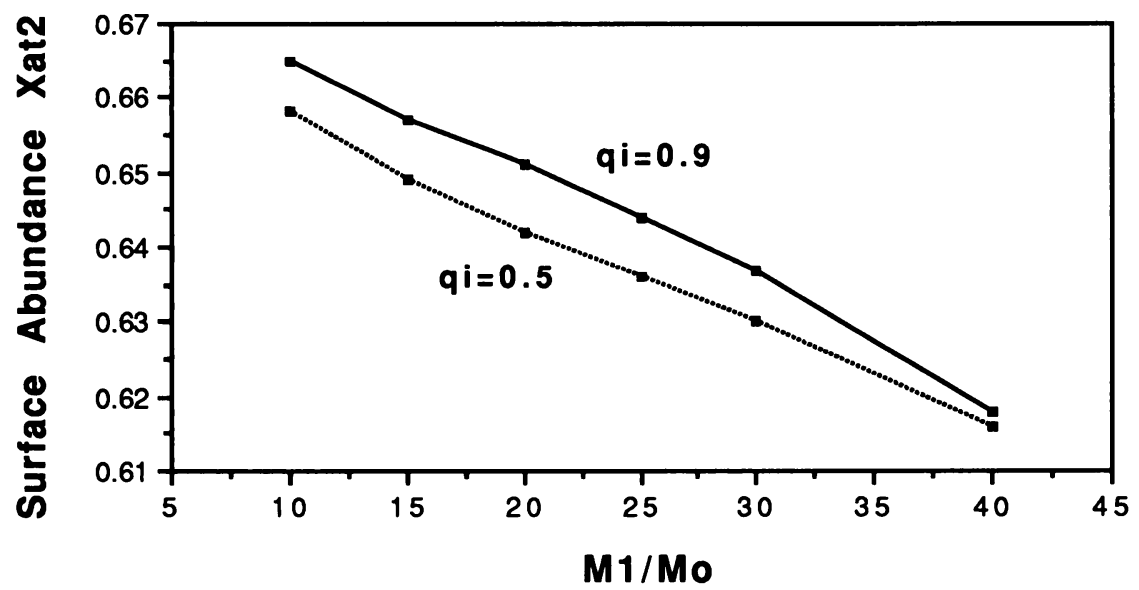

Figure 4. Hydrogen abundance by mass at the surface of the gainer, after case B of mass transfer and thermohaline mixing of the accreted layers that are affected by CNO burning, as a function of initial mass of the mass losing star. The results are given for 2 values of the initial mass ratio. 
Indeed, Howarth and Prinja (1989) tried to find a dependence of the stellar wind mass loss rate on rotation (see also Marlborough and Zamir, 1984, Pauldrach et al. 1986). From their observations they derived the relation

$$
\Delta \log M=(0.8 \pm 0.6)\left[v_{\text {eq }} \sin \mathrm{i} / \mathrm{v}_{\text {break-up}}-0.24\right]
$$

Also Blomme (1991) investigated the same influence on the results for a radiation driven wind. Both investigations lead to a rather small increase in the mass loss rate (20 to $50 \%$ at the equator)

Thus, in order for the binary hypothesis to survive, additional mixing is needed, bringing more detoriated material to the surface, or a substantial difference in physical structure must exist between accretion stars and normal main sequence stars.

The second hypothesis deserves somewhat more attention. Are accretion stars really alike main sequence stars after RLOF ?

\section{Are accretion stars alike main sequence stars ?}

Analysis of observations already points to a fargoing similarity between normal main sequence stars and bright components in semidetached systems (Giuricin and Mardirossian, 1981 ; De Greve, 1986 ; Hilditch and Bell, 1987 ; Garcia and Gimenez, 1990)

For the sake of simplicity, we focus our attention on masses around $15 \mathrm{M}_{0}$. We will consider first an accretion star of $15.2 \mathrm{M}_{0}$, resulting from an early case $\mathrm{B}$ of mass transfer of $12 \mathrm{M}_{\mathrm{o}}+10.8 \mathrm{M}_{0}$.

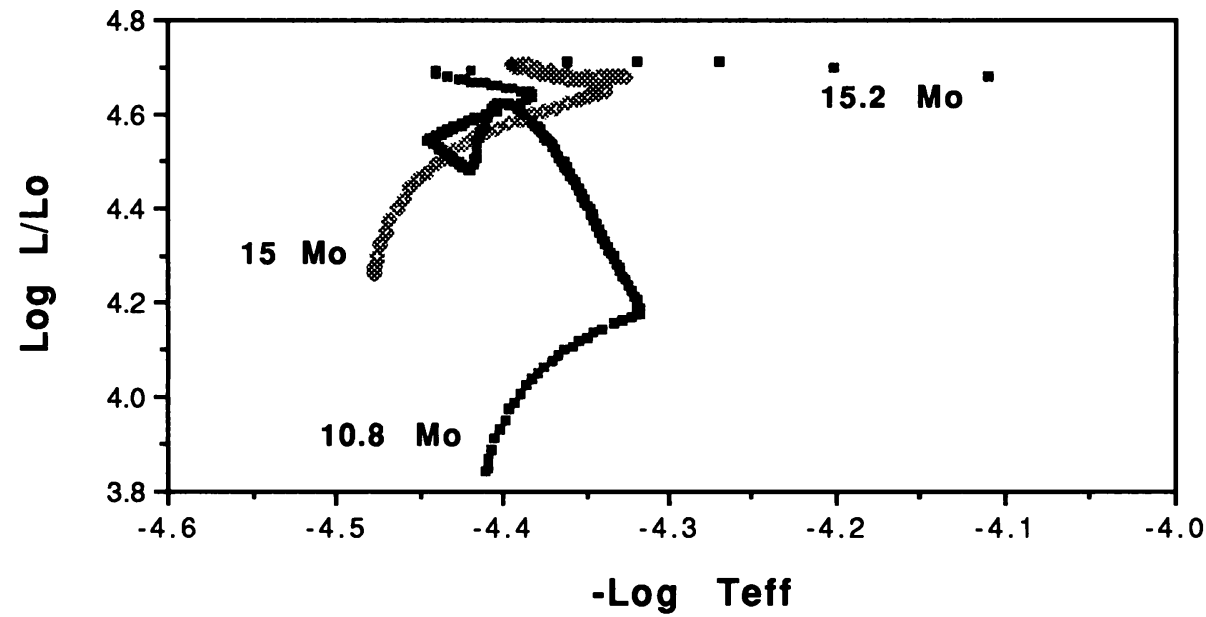

Figure 5. HRD of the secondary component of the system $12 \mathrm{M}_{\mathrm{O}}+10.8 \mathrm{M}_{\mathrm{O}}$, before, during and after a case B of mass transfer (full squares). The track of a single star of 15 $\mathbf{M}_{\mathbf{O}}$ (open diamonds) is given for comparison. 
At the onset of mass exchange, the secondary is a main sequence star with central hydrogen content $X_{C}=0.15$. After mass transfer (with $B=0.5$ ) a bright massive star results, with mass $15.2 \mathrm{M}_{0}$ and $\mathrm{X}_{\mathrm{c}}=0.31$. In the HRD the star is located nearly on the evolutionary track of a single star of mass $15 \mathrm{M}_{0}$ (Figure 5). But also the inner structure agrees almost completely with that of a normal main sequence star (Figure 6), the only exception being a little less hydrogen in the outer envelope $\left(X_{\mathrm{at}}=0.67\right)$. It is clear that such a star will further follow an identical evolution as a single star.

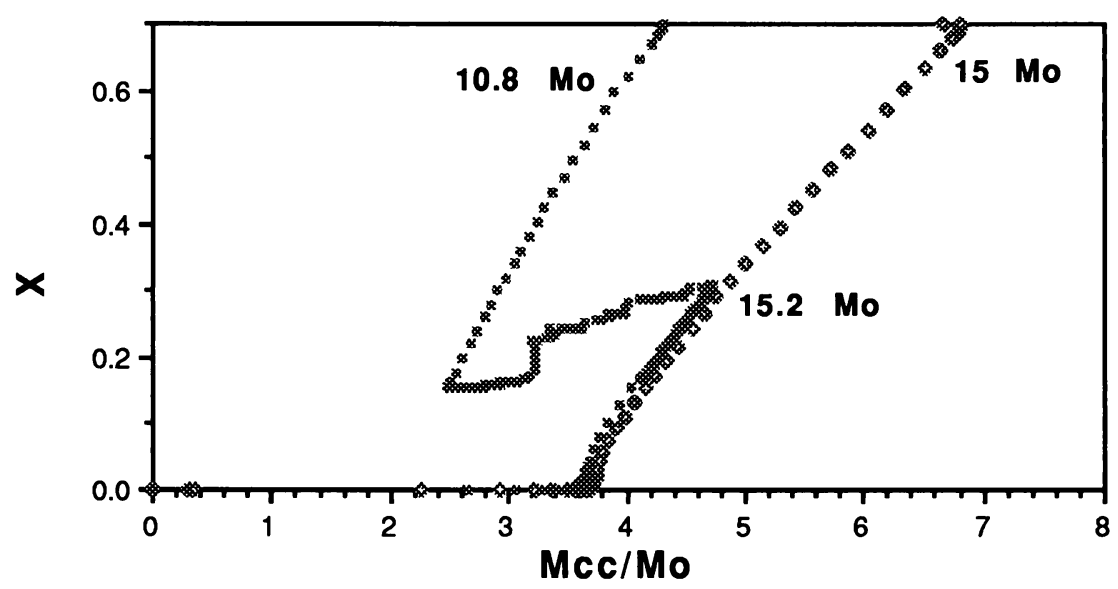

Figure 6. Hydrogen content by mass as a function of the convective core mass, for the secondary of $12 \mathrm{M}_{\mathrm{O}}+10.8 \mathrm{M}_{\mathrm{O}}$, before, during and after a case $\mathrm{B}$ of mass transfer (full squares). The evolution for a single star of $15 \mathrm{M}_{\mathrm{o}}$ is given for comparison (open diamonds).

Let us now consider a system with a mass ratio almost equal to $1(\sim 0.99), M_{1}=9 M_{0}$ and initial period of 12 days (de Loore and De Greve, 1991). The fundamental difference with the foregoing system is that at the time the mass transfer sets in, hydrogen is also exhausted in the core of the secondary component (Podsiadlowski et al., 1990) This has farreaching consequences for the evolution of the secondary after RLOF (in this case, calculated with $B=1$ ). After RLOF the accretion star is found in the main sequence band, roughly at the luminosity of a star with the same mass. However, the central region of this star is completely different from a main sequence star. The frozen-in hydrogen profile is still that of the initial mass. As a result, a smaller helium core develops (Figure 7), a smaller $\mathrm{H}$-shell region (with varying $\mathrm{H}$-content), and a much larger homogeneous zone. The H-shell produces less energy (as does the helium core), forcing the star to keep its radius small (to maintain its luminosity). In the HRD, the star does not evolve towards the red. It spends its H-shell and core helium shell burning in or close to the main sequence band, with $\log$ Teff varying from 4.41 to 4.10 , hence between spectral types B0.5 and B6-8 (Figure 8, see also the poster of Joss et al.).

The essential condition for such an evolution is that the secondary terminates its core hydrogen burning before the start of the RLOF (this requires a large mass ratio and a large period) : 
$\begin{array}{ll} & t_{H 2}<t_{H 1}+\Delta t_{H S 1} \\ \text { with } & t_{H}=\text { timescale of core hydrogen burning }\end{array}$

$\Delta t_{H S}=$ timescale of H-shell burning till the beginning of RLOF.

In practice this leads to periods of some tens of days and mass ratios of 0.98 or more. If this is part or whole of the explanation of the excess of yellow supergiants in the LMC, then we still have to find out what is going on in our own galaxy.

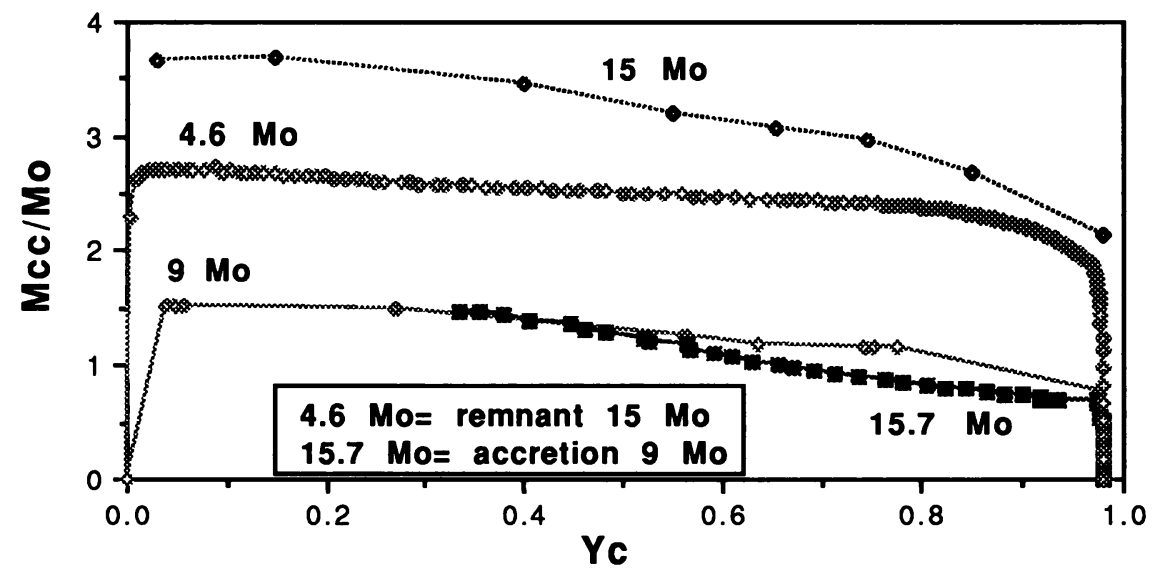

Figure 7. Evolution of the convective core during core helium burning, as a function the central helium content. The upper curve shows the evolution for a single star of $15 \mathbf{M}_{0}$, the 4.6 $\mathrm{M}_{\mathrm{o}}$ curve is that of its remnant after a case B of mass transfer. The lower curve (filled squares) is that of the secondary component of the system $9 \mathrm{M}_{0}+8.98 \mathrm{M}_{0}$, after accretion up to a mass of $15.7 \mathrm{M}_{0}$. The curve for a single star of $9 \mathbf{M}_{0}$ is also given.

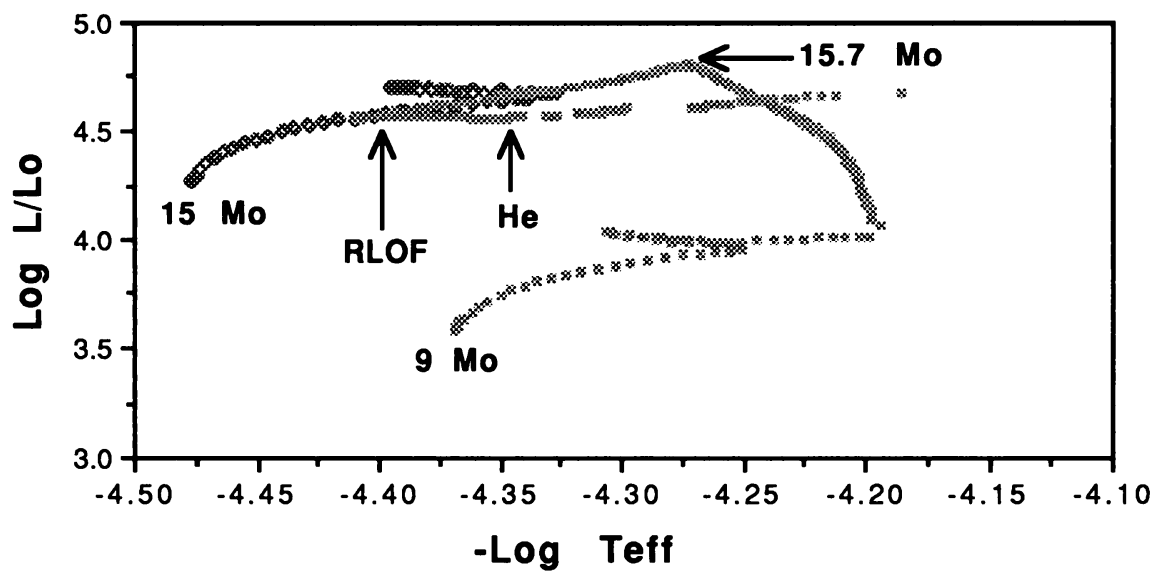

Figure 8. HRD of the secondary component of the system $9 \mathrm{M}_{0}+8.98 \mathrm{M}_{0}$, before, during and after a case B of mass transfer (full squares). The track of a single star of 15 $M_{0}$ (open diamonds) is given for comparison. The end of the RLOF (RLOF) and the onset of helium burning $(\mathrm{He})$ are indicated. 
Conclusion :

Mass transfer leads to two different types of "post-ms" giants and supergiants. Mass transfer starting while the secondary is still in the phase of core hydrogen burning, results in bright stars that in all aspects evolve like single stars of comparable mass. Stars that accrete mass during $\mathrm{H}$-shell burning, do not adapt their inner structrure to the new mass. They produce less energy and remain in or close to the main sequence band for the rest of their life.

\section{Acknowledgement.}

I thank the Scientific Committee of this Symposium for the invitation to present this review. The realisation of it was financed by grants S2/5 E96 (National Fund of Scientific Research of Belgium) and grant SCI-0065-C (EC-SCIENCE program).

\section{References}

Bell, R.A., Hill, G., Hilditch, R.W., Clausen, J.V., Reynolds, A.P., Gimenez, A. : 1991, Mon. Not. R. astr. Soc. 250, 119

Blomme, R. : 1991, Ph. D. thesis, V.U.B. Brussels

De Greve, J.P. : 1986, Space Sci. Rev. 43, 139

De Greve, J.P., Cugier, H. : 1989, Astron. Astrophys. 211, 356

de Loore, C., De Greve, J.P.:1991, poster, this volume

de Loore, C., De Greve, J.P.:1991, Astron. Astrophys. Suppl. Ser. (in press)

Figueiredo, J., De Greve, J.P., de Loore, C. : 1991, Astron. Astrophys. (in press)

Fitzpatrick, E.L., Garmany, C.D. : 1990, Astrophys. J. 363, 119

Garcia, J.M., Gimenez, A. : 1990, Astrophys. Space Sci. 169, 121

Giuricin, G., Mardinossian, F. : 1981, Astrophys. J. Suppl. Ser. 46, 1

Hilditch, R.W., Bell, S.A. : 1987, Mon. Not. R. astr. Soc. 229, 529

Howarth, I.D., Prinja, R.K.: 1989, Astrophys. J. Suppl. Ser. 69, 527

Huang, R.Q., Taam, R.E. : 1990, Astron. Astrophys. 236, 107

Joss, P.C., Hsu, J.J.L., Podsiadlowski, P.: 1991, poster, this volume

Kippenhahn, R., Ruschenplatt, G., Thomas, H.-C.: 1980, Astron. Astrophys. 91, 175

Kudritzki, R et al. : 1989, Proc. IAU Coll. 113

Livio, M., Soker, N. : 1988, Astrophys. J. 329, 764

Marlborough, J.M., Zamir, M.: 1984, Astrophys. J. 276, 706

Michaud, G., Dupuis, J., Fontaine, G., Montmerle, T. : 1987, Astrophys. J. 322, 302

Packet, W. : 1988, Ph. D. thesis, V.U.B. Brussels

Paczynski, B. : 1991, Astrophys. J. 370, 597

Pauldrach, A., Puls, J., Kudritzki, R.P.: 1986, Astron. Astrophys. 164, 86

Podsiadlowski, P, Joss, P.C., Rappaport, S.: 1990, Astron. Astrophys. 227, L9

Popham, R., Narayan, R. : 1991, Astrophys. J. 370, 604

Proffitt, C.R., Michaud, G. : 1989, Astrophys. J. 345, 998

Ray, A., Rathnasree, N. : 1991, Mon Not. R. astr. Soc. 250, 453

Tuchman, R., Wheeler, J.C. : 1990, Astrophys. J. 363, 255

Tutukov, A., Yungelson, L.: 1991, Astron. Zh. 71 (in press)

Van Hamme, W., Wilson, R.E. : 1990, Astron. J. 100, 1981

Vrancken, M., De Greve, J.P., Yungelson, L., Tutukov, A. : 1991, Astron. Astrophys. (in press)

Yungelson, L., Tutukov, A. : 1991, in K.A. van der Hucht and B. Hidayat (eds.), Wolf-Rayet Stars and Interrelations with Other Massive Stars in Galaxies, Kluwer Co., $\quad$ p. 459 


\title{
ZETA AURIGAE TYPE BINARIES
}

\author{
KENNETH G. CARPENTER \\ Laboratory for Astronomy and Solar Physics \\ NASA - Goddard Space Flight Center \\ Greenbelt, MD 20771
}

\begin{abstract}
.
Recent progress in the study of $\zeta$ Aur and VV Cep systems is reviewed, with an emphasis on what has been learned about the extended atmospheres and winds around the cooler components.

Key words: Stars: eclipsing binaries - Stars: chromospheres - stars: late-type - stars: winds
\end{abstract}

\section{Introduction}

$\zeta$ Aur binaries are interacting binary systems with both a hot and a cool component, in which the hot component is eclipsed by the extended atmosphere of the cool component. Their spectra are marvelously complicated: below about $2800 \AA$, the spectra is that of a B-star photosphere on which wind, chromospheric, shock, accretion, and interstellar lines appear superposed. Above $2800 \AA$, the photosphere of the cool star is visible as well, making optical work very entertaining and challenging. In the 'classical' $\zeta$ Aur systems, the cool star is a supergiant, but the class now contains examples where the cool star is a giant. For the purposes of this review, I take this class to include the VV Cep systems, in which the primary is an M-star, as well as the $\zeta$ Aur systems themselves, in which the primary is a $\mathrm{G}$ or $\mathrm{K}$ star.

These stellar systems are of interest for two reasons: 1) for the interactions between the components and 2) for the marvelous opportunity they provide us for the study of the extended atmosphere of the cool components. The visible interactions include shocks and accretion as well as an increased ionization of the cool wind by radiation from the hot star. Eclipses of the hot star by the atmosphere of the cool star allow us to study the height dependence of the thermodynamic parameters (e.g. excitation, ionization, density, temperature, flow and turbulent velocities) of the latter's chromosphere and stellar wind.

In this review I will summarize the most recent work on $\zeta$ Aur systems, concentrating on the very numerous investigations of the extended outer atmospheres of the cool components. The reader is referred to a rich literature of reviews on this topic for further information, particularly on earlier investigations. Some of the major reviews include Schneller (1935), Wellmann (1939), Wilson (1960), Wright (1970), Sahade and Wood (1978), Hack and Stickland (1987), Reimers (1989), and Guinan (1990).

\section{Membership}

Members of the $\zeta$ Aur -type systems which have been well-studied are listed below: 
- Zeta Aurigae Systems (G-K giant/supergiant with hot dwarf companion)

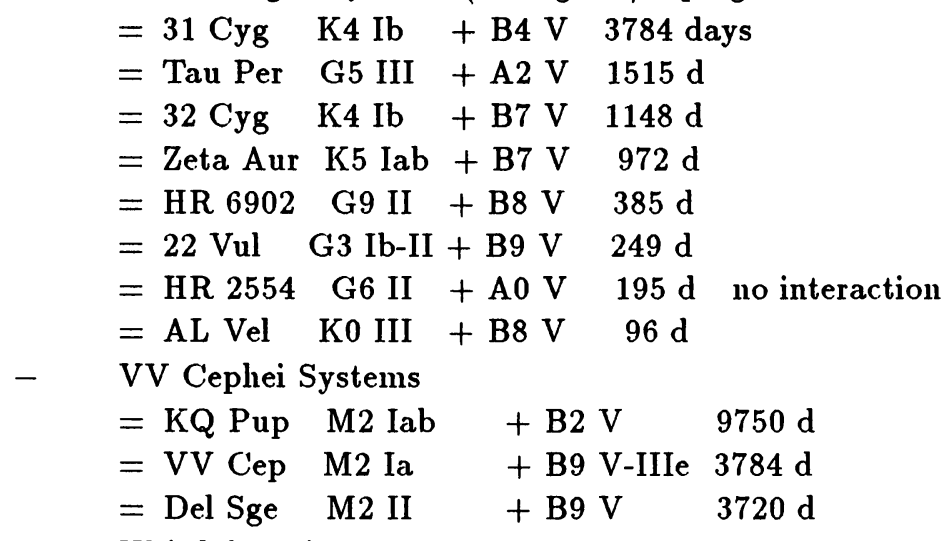

- Weird \& Unique

= Eps Aur F0 Iab + disk ?? 9890 days (primary is lightbulb)

Only true eclipsing systems have been included in the above list. Epsilon Aur is in a separate class all by itself and easily warrants its own review. It will not be considered further in this one.

\section{Interactions}

There are two major types of interactions which occur in $\zeta$ Aur systems: the formation of shocks and accretion disks as the B-star moves supersonically through the cool wind, and the more remote effects on cool star wind due to the presence of the hot star such as gravitational perturbations and ionization by radiation from the B-star.

\subsection{Shocks and ACCretion Disks}

Chapman (1981) is credited with the discovery of accretion shocks in the UV. The lines formed include resonance lines of C IV, Si IV, Al II, N V, and excited Fe III (34) and are seen variously as very broad absorption, regular or inverse $\mathrm{P}$ Cyg, or pure emission profiles. The emitting region clearly moves with the B-star. Che-Bolnnenstengel and Reimers (1986) demonstrated that the geometric size of the accretion shock is small compared to the size of the circumstellar envelope and can thus be neglected in wind analysis.

Ahmad (1989) has recently shown that 31 Cyg has an intermediate severity shock in comparison with other $\zeta$ Aur systems. He notes that $\zeta$ Aur and $22 \mathrm{Vul}$ have strong reverse $\mathrm{P}$-Cyg profiles in their high-temperature lines and show high-velocity absorption by C IV and N V, while 31 Cyg shows only weak reverse P-Cyg profiles that are visible only at angles near that of the shock cone and absorption in $\mathrm{C} I V$, but not in N V. 32 Cyg shows no reverse P-Cyg profiles and no C. IV/N V absorption. He further notes that the $82 \mathrm{~km} / \mathrm{s}$ Fe II and Mg II absorption components are circumstellar in origin and consistent with the wind terminal velocity of $80 \mathrm{~km} / \mathrm{s}$. 
Broad or double-peaked emission is taken as the possible signature of an accretion disk. The latter has been seen in $\delta$ Sge by Reimers and Schroder (1983), while Che-Bohnenstengel and Reimers (1986) report signs of an accretion disk in $\zeta$ Aur and $\delta$ Sge.

\subsection{Effects on Cool Wind}

More remote effects of the B-star on the wind of cool component include the influence of the gravitational field of the hot star on the latter's wind and flow patterns and perhaps more significantly the ionization of the cool wind by the radiation from the B-star. This radiation creates an H II region around the B-star and inside the cool star envelope and certainly affects mass-loss estimates for ions such as $\mathrm{Fe}, \mathrm{Si}$, etc.

\section{Probing the Winds of Cool Giants and Supergiants in $\zeta$ Aur Systems}

One of the most important characteristics of $\zeta$ Aur systems is that they allow us to probe the height dependence of the thermodynamic parameters of the outer atmospheres of cool, high-luminosity stars. This information can be used to increase our understanding of the structure of these outer atmospheres and to constrain models of chromospheric heating and wind-driving mechanisms. The cool winds and chromospheres are visible through both emission and absorption lines. Hempe and Reimers (1982) summarize the characteristics of the wind lines of Fe II, Si II, Al II, Mg II, S II, and O I: 1) they have P Cyg profiles in all phases, except that they go into pure emission during totality (resonance scattering of B-star photons), 2) Fe II (UV 9) however remains in pure absorption, due to the strong emission from UV 191, and allows a COG analysis during all phases, and 3) chromospheric lines are seen near totality as narrow absorption lines at zero velocity.

UV and optical spectra each have advantages and disadvantages, but provide very nice complementary information, if both are available for the same object. UV spectra suffer minimal contamination by the cool star, have a well-defined continuum over which resonance-scattered wind lines can be easily studied, contain strong lines of all common chromospheric species, and allow an examination of the chromosphere out to 1-2 stellar radii. However, the UV spectrum is very crowded with lines and analysis can be difficult. Bauer and Stencel (1989) provide a very handy atlas of the $31 \mathrm{Cyg}$ UV spectra, complete with line identifications. Optical spectra suffer from a dual continuum, but have much less crowded absorption features, so that weak lines are more easily measured. More neutrals are available and lower layers of the chromosphere can be studied than in the UV. It is especially useful to have the Balmer series observed since that provides critical modeling information on the hydrogen ionization. VV Cep systems are in general more difficult to analyze than $\zeta$ Aur systems since the optical spectrum is more complex and the M-supergiant has strong emissions in the UV. 


\subsection{Results from Curve-of-Growth Techniques}

Computer codes to handle the non-spherically symmetric line transfer needed to model the profiles have been developed by Hempe (1982) and Baade $(1986,1990)$. Hempe used a 2-level escape probability approximation for the source function to get mass-loss rate estimates. Baade (1986) validated these results with a multi-level modification, although neither calculation matches profiles exactly at all phases.

Measurements of the wind velocity, turbulent velocity and mass-loss rate have been published by Che et al. (1983) for $\zeta$ Aur , $31 \mathrm{Cyg}$, and $32 \mathrm{Cyg}$, while Reimers and Che-Bohnenstengel (1986) have measured $v_{\text {win. }}=170 \mathrm{~km} / \mathrm{s}$ for $22 \mathrm{Vul}$. Reimers (1989) summarizes turbulence and wind velocity measurements for $\zeta$ Aur systems and notes that the turbulent velocity is, in general, approximately one-half of the wind velocity. Baade (1990) uses a '2-integral operator' to get a better 32 Cyg model in which $\dot{M}=1.5 \times 10^{-8}$ solar masses $/ \mathrm{yr}, \mathrm{v}_{\text {wind }}=90 \mathrm{~km} / \mathrm{s}$, and $\mathrm{v}_{\text {turb }}=30$ $\mathrm{km} / \mathrm{s}$.

Measurements of the density and wind velocity versus height in $\zeta$ Aur systems have been given by Schroder $(1985,1986)$ and, for 22 Vul, Schroder and Reimers (1989). In the first paper, the density results indicate the observed acceleration takes place at greater heights and is more gradual than would be the case for Alfven-driven winds. The second paper gives equations for these parameters based on a COG analysis, the equation of continuity, and the assumption of a steady wind.

Temperature measurements from observed populations of Fe II levels have been derived by Che-Bohnenstengel (1984) for $32 \mathrm{Cyg}\left(\mathrm{T}_{e}=4800 \mathrm{~K}\right.$ for $\mathbf{n}_{e}=0.01$, $10000 \mathrm{~K}$ for smaller electron densities), and by Reimers and C'he-Bohnenstengel (1986) for $22 \mathrm{Vul}(20000-40000 \mathrm{~K})$. Schroder (1986)measured a variation in temperature from 8500 to $11000 \mathrm{~K}$ over the region from $0.2 \mathrm{R}_{*}$ to $0.5 \mathrm{R}_{*}$ and inferred that heating occurs where the wind starts.

\subsection{The Transition from Coronae to Cool Winds}

Schroder (1990) has presented a major study of the physical state of supergiant chromospheres in the region of the HR diagram where the transition from coronae to cool winds occurs. He presents direct evidence for a new type of hot circumstellar matter in HR 6902 and HR 2554 and infers that these are red giant stars with a solar-type 'hot' wind but a 'cool' corona. The latter has transition-region-like temperatures, but is extended like a corona. HR 6902 is to the right of the $\mathrm{Mg}$ II dividing line $(\mathrm{V} / \mathrm{R}=0.75)$, but has a highly ionized, high speed, low mass wind $\left(120 \mathrm{~km} / \mathrm{s}, 82000 \mathrm{~K}, \dot{M}=1.5 \times 10^{-11}\right.$ solar masses/year $)$. It is difficult to know how a star such as this relates to the hybrid single stars, because a major signature of the hybrid phenomenon is the presence of C IV and Si IV emission, which in $\zeta$ Aur systems can be caused by binary interactions. Schroder suggests that the hybrid stars occupy the region between HR 6902 and $22 \mathrm{Vul}$ and have radially-diverted wind regions alternately filled with cool and hot matter. He also identifies a logical sequence, which he believes is an ionization effect, for four critical systems, in terms of distance from the dividing line: $22 \mathrm{Vul}$ (neutral extended chromosphere), 
HR 6902 (cool, inner layer, extended but decreased (by a factor of $10^{3}$ ) Fe II absorption and hot circumstellar matter further out), HR 2554 (no dense inner layer, but extended/decreased Fe II absorption and hot circumstellar matter), and $\tau$ Per (decreased Fe II absorption from a thin, low layer only).

\subsection{Recent Progress in Optical Spectroscopy}

Recently, Griffen et al. (1990) defined a method of spectral subtraction with which they are able to isolate the 3650 - $4650 \AA$ spectra of the B-star and the K-star chromosphere in $\zeta$ Aur for the first time. Using optical spectra from the 1987 eclipse of $\zeta$ Aur and this technique, they identified over 260 absorption features from the chromosphere and produced a model for the geometry of the eclipse. Their results indicate that, if the lower chromosphere rotates rigidly with the supergiant, the rotation period is synchronous with the orbital period.

Using the data and spectral subtraction technique from the Griffen et al. (1990) paper, Schroder et al. (1990) produced an empirical model of the lower chromospheric layers of the $\zeta$ Aur primary. They applied a curve-of-growth analysis of the chromospheric absorption spectrum to derive density vs. height for various atomic species, to estimate the ionization ratios of $\mathrm{Fe} \mathrm{II} / \mathrm{Fe} \mathrm{I}$ and $\mathrm{Mg} \mathrm{II} / \mathrm{Mg} \mathrm{I}$, and to derive the electron density and temperature and hydrogen ionization at $2.3 \times 10^{6}$ and $6.1 \times 10^{6} \mathrm{~km}$. They found $\log \left(\mathrm{N}_{e}\right)=9.6$ and 9.2 , respectively, and $\mathrm{T}_{e}=5500 \mathrm{~K}$ at both heights, while hydrogen was found to be $1.8 \%$ and $5 \%$ ionized at the two heights. These results nicely complement IUE results which apply only to higher layers of the chromosphere.

\subsection{Results from Comparing Synthetic Spectra to Observations}

Eaton has taken another approach to the study of $\zeta$ Aur systems, by attempting to match broad regions of UV and optical observational and synthetic spectra to determine the chromospheric ionizations, column densities, temperatures, and turbulent velocities. Eaton (1988) defines the procedure and applies it to $31 \mathrm{Cyg}$, while Eaton (1991) presents results for $\zeta$ Aur itself. He 'fudges' NLTE effects and uses the LTE Kurucz (1979) model atmosphere program ATLAS to produce the synthetic spectra and derive the thermodynamic parameters. The correct ionizations states are forced by restricting the stages allowed and the correct electron density is forced by altering the effective gravity. Hydrogen ionization is derived from the Balmer lines, while $\mathrm{Ca}$ II is used to infer the calcium ionization and the electron density.

The Lyman-alpha column density is derived assuming hydrogen is mostly neutral and metals mostly ionized. Analysis of Fe II, Cr II, Si II column densities give the same results and also agree within a factor of two with the Schroder (1985) results on $\zeta$ Aur. He points out that the Balmer lines are seen to 100 solar radii, while the UV lines are seen to $200-370$ solar radii. His results are similar to semiempirical models based on emission lines in that the temperature rise is the same, the turbulent velocity is about $2-3 x$ the sound speed, and there is no hydrostatic equilibrium vs. the gas pressure. Eaton also believes that the chromosphere is confined by magnetic fields, supported by the turbulent pressure of Alfven waves and 
that the turbulent pressure is sufficient to support the atmosphere. He finds that the electron density remains constant with height $\left(\approx 10^{\circ}\right)$ and infers that the ionization of hydrogen must rise with height to maintain the density at a constant value.

For 31 Cyg, Eaton (1988) finds that hydrogen is essentially neutral, while the metals are 10000:1 singly-ionized:neutral. He finds a temperature range versus radius of $5000-14,400 \mathrm{~K}$, with a mass-averaged value of $6400 \mathrm{~K}$, a turbulent velocity of $20-25 \mathrm{~km} / \mathrm{sec}$, and abundances which agree with Luck and Lambert (1985).

\subsection{Semi-Empirical Wind Model}

Kuin and Ahmad (1989) have published a new semi-empirical wind model of $\zeta$ Aur systems based on observations of 31 and $32 \mathrm{Cyg}$ and $22 \mathrm{Vul}$. The velocity function, non-thermal velocity, and upper limits on $\mathrm{N}_{e} / \mathrm{N}_{H}$ are as inferred from observations by Schroder (1985), but the terminal velocity is taken from Ahmad and Stencel (1988). They assume that the energy fluxes are supplied by Alfven waves (since acoustic wave fluxes are too low based on observed line broadening), and a radially symmetric, steady outflow, and then derive constraints on damping length to find $\mathrm{T}_{e}$ and $\mathrm{N}_{e}$. The resulting model describes the supergiant wind $\mathrm{OK}$, including the ionization behavior. They find that they need a lower limit on $\dot{M}$ in order to get a cool wind, i.e. that if there is too little mass, the radiative losses don't balance the heating and the outer atmosphere warms up. Finally, they find that the damping scale length of the waves must be small near the star and larger further out.

\subsection{A First Look at a $\zeta$ Aur System with a Ko III Primary}

Eaton et al. (1990) have taken advantage of the unusual chance, provided by the $\mathrm{AL}$ Vel system, to use the binary technique on a giant (rather than a supergiant) star. The AL Vel system consists of a K0 III primary and a B8 V secondary. The latter allows us to probe the chromosphere out to about 2 stellar radii. Two eclipses in this system have been observed with IUE and although the wind lines are saturated in the inner regions, data on the upper chromosphere $(0.7$ and 2.0 stellar radii) are good. This study indicates that common ions are singly-ionized, except in a region near the B-star where the common ions are doubly-ionized and that the excitation temperature inferred from Fe II lines is in the range from 10000 $15000 \mathrm{~K}$. The presence of high ionization lines during totality imply the existence of an accretion disk or shell. Analysis of the $\mathrm{Mg}$ II P Cyg profiles indicates a wind expansion of about $60 \mathrm{~km} / \mathrm{s}$, while the width of the emission lines implies a corotation of the wind out to $9 \mathrm{~K}$-star radii.

\section{First Interferometry of $\zeta$ Aur Systems}

The first interferometric resolution of $\zeta$ Aur , 31 Cyg, and $32 \mathrm{Cyg}$, using the I2T CERGA interferometer in the near-IR has been reported by Benedetto and Ferluga (1990), who give angular diameters of $5.5 \pm 0.3,5.7 \pm 0.6$, and $5.5 \pm 0.5$ milliarcseconds, respectively. Combining these results with eclipse timing and orbital velocity information, they derive radii of $154 \pm 13,169 \pm 20$, and $215 \pm 33$ solar radii, and 
distances of $260 \pm 28,282 \pm 23$, and $364 \pm 55$ parsecs, respectively. Based on these results, they conclude that the companion of $\zeta$ Aur is already evolving to luminosity class IV.

\section{VV Cephei Systems}

The analysis of VV Cephei systems has proceeded more slowly than that of $\zeta$ Aur systems since the spectra of the hot and cool components are more difficult to disentangle, but several interesting papers have appeared in the last several years. Hack, Engin and Yilmaz (1989) have studied IUE data on VV Cep taken over the period from 1978-1984. They report that no interstellar absorption is seen, but derive an $\mathrm{E}(\mathrm{B}-\mathrm{V})=\mathbf{0 . 4 0}$, a value which is larger than that derived from optical data. They estimate the spectral type of the companion as A0 II and report that the UV spectrum is mostly absorption, in contrast to the optical spectrum which has lots of strong emission, although UV emission is seen from N I, O I, Mg II, Fe II, and weakly from Lyman $\alpha$. They present a summary of the UV multiplets seen in the spectrum, but no detailed list of the identified lines.

Recent results from the Goddard High Resolution Spectrograph (GHRS) on Hubble Space Telescope may help to explain the huge difference in O I (UV 2) flux seen from VV Cep and $\alpha$ Ori. Hack and Stickland (1987) have pointed out that it is nearly 3000x brighter in VV Cep than in $\alpha$ Ori. Carpenter et al. (1991) have shown, using GHRS spectra, that the large deficiency in observed O I (UV 2) flux from $\alpha$ Ori, relative to the 'typical' late-type star, is apparently due to strong circumstellar absorption by the $(9,0)$ band of the 4th Positive System of CO. Presumably, the circumstellar environment of VV Cep is much warmer due to the presence of the B-star and much less conducive to the formation of $\mathrm{CO}$.

Altamore et al. (1991) present for the star KQ Pup (M1-2 Iab + Be, 26.7 year period) an atlas of IUE high-dispersion, DAO Coude, and ESO spectra which cover the wavelength regions $1223-3228,3180-5160$, and $6540-6590 \AA$ and state that digitized spectra are available upon request.

Rossi et al. (1991) analyze Coude and CAT CES optical spectra of KQ Pup taken over the period 1969-1984 and compare them with IUE spectra taken in 1979. They note that the optical spectrum is that of an M-star photosphere on top of which is emission from the wind regions ionized by the B-star from the ions [S II], [Cu], [Fe II], Fe II, and [Ni II]. The resonance lines and excited lines of doubly-ionized metals are broad and red-shifted by $37 \mathrm{~km} / \mathrm{s}$, relative to the radial velocity of the star. The authors infer from this that the lines are formed in opaque turbulent matter flowing near and downward to the star. They prepare from these data a model in which the emission comes from a cool wind region ionized by the B-star and from a shock at the interface of the hot and cool winds.

\section{Summary}

The capability which $\zeta$ Aur systems provide us for resolving the structure of the extended atmospheres of cool giant and supergiant stars has been agressively exploited in recent years, both in the UV using IUE (Schroder 1990, Baade 1990, Kuin 
and Almad 1989) and from the ground with more sophisticated analysis of optical data (Griffen et al. 1990, Schroder et al. 1990). Eaton $(1988,1991)$ has used both in comparisons with extensive synthetic spectra in a different, but equally promising approach to the study of these systems. The $\zeta$ Aur class has been expanded to include several systems with a class III giant, and the initial study of one of them (AL Vel) by Eaton et al. (1990) indicates that much can be learned about the atmospheres of giant stars as well using the 'binary technique'. The first interferometric resolution of $\zeta$ Aur systems (Benedetto and Ferluga 1990) has provided us with exciting basic information on them, including radii and distances. Detailed work on the VV Cep system KQ Pup has been pursued by Altamore et al. (1991) and Rossi et al. (1991) using both UV and optical spectra, with the latter providing a model for the origin of emission lines in that system. It is clear we can look forward to many more years of exciting developments in the study of $\zeta$ Aur systems using ground-based data and space-based UV spectra from IUE and HST.

\section{References}

1. Ahmad, I. A. 1989, ApJ, 338, 1011.

2. Ahmad, I. A. and Stencel, R. E. 1988, ApJ, 123, 1234.

3. Altamore, A., Rossi, C., and Viotti, R. 1991, A\&AS, submitted.

4. Baade, R. 1986, A\&A, 154, 145.

5. Baade, R. 1990, in 'Evolution in Astrophysics', ed. E. J. Rolfe, ESA SP-310, p. 65.

6. Bauer, W. H. and Stencel, R. E. 1989, ApJS, 69, 667.

7. Benedetto, G. P. and Ferluga, S. 1990, A\&A, 236, 449.

8. Carpenter, K. G., Robinson, R. D., Ebbets, D. C., Brown, A., and Linsky, J. L. 1991, BAAS, 23, 910.

9. Chapman, R. D. 1981, ApJ, 248, 1043.

10. Che-Bonenstengal, A. 1984, A\&A, 138, 333.

11. Che-Bonenstengal, A. Hempe, K., and Reimers, D. 1983, A\&A, 126, 225.

12. Che-Bonenstengal, A. and Reimers, D. 1986, A\&A, 156, 172.

13. Eaton, J. A. 1988, ApJ, 333, 288.

14. Eaton, J. A. 1991, ApJ, submitted.

15. Eaton, J. A., Kondo, Y., McCluskey, G. F., and Shore, S. 1990, AJ, 100, 799.

16. Griffen, R. E. M., Griffen, R. F., Schroder, K.-P., and Reimers, D. 1990, A\&A, 234, 284.

17. Guinan, E. 1990, in 'Evolution in Astrophysics', ed. E. J. Rolfe, ESA SP-310, p. 74.

18. Hack, M., Engin, S. and Yilmaz, N. 1989, A\&A, 225, 143.

19. Hack, M. and Stickland, D. 1987, in 'Scientific Accomplishments of IUE', ed. Y. Kondo, Reidel, NY, NY, p. 445.

20. Hempe, K. 1982, A\&A, 115, 133.

21. Hempe, K. and Reimers, D. 1982, A\&A, 107, 36.

22. Kuin, N. P. M. and Ahmad, I. A. 1989, ApJ, 344, 856.

23. Kurucz, R. L. 1979, ApJS, 40, 1.

24. Luck, R. E. and Lambert, D. L. 1985, ApJ, 298, 782. 
25. Reimers, D. 1989, in 'FGK Stars and T Tauri Stars', eds. L. Cram and L. Kuhi, NASA SP-502, p. 53.

26. Reimers, D. and Che-Bonenstengal, A. 1986, A\&A, 166, 252.

27. Reimers, D. and Schroder, K.-P. 1983, A\&A, 124, 241.

28. Rossi, C., Altamore, A., Baratta, G. B., Friedjung, M., and Viotti, R. 1991, A\&AS, submitted.

29. Sahade, J. and Wood, F. B. 1978, in 'Interacting Binary Stars', Pergammon, Oxford, p. 121.

30. Schneller, H. 1935, Sterne, 15, 185.

31. Schroder, K.-P. 1985, A\&A, 147, 103.

32. Schroder, K.-P. 1986, A\&A, 170, 70.

33. Schroder, K.-P. 1990, A\&A, 236, 165.

34. Schroder, K.-P. and Reimers, D. 1989, A\&A, 208, 223.

35. Schroder, K.-P., Griffen, R. E. M., and Griffen, R. F. 1990, A\&A, 234, 299.

36. Wellman, P. 1939, Veroff. Univ. Berlin-Babelsberg, 12, No. 4.

37. Wilson, O. C. 1960, in 'Stellar Atmospheres', ed. J. L. Greenstein, Univ. of Chicago press, Chicago, p. 436.

38. Wright, K. O. 1970, Vistas in Astronomy, 12, 147. 